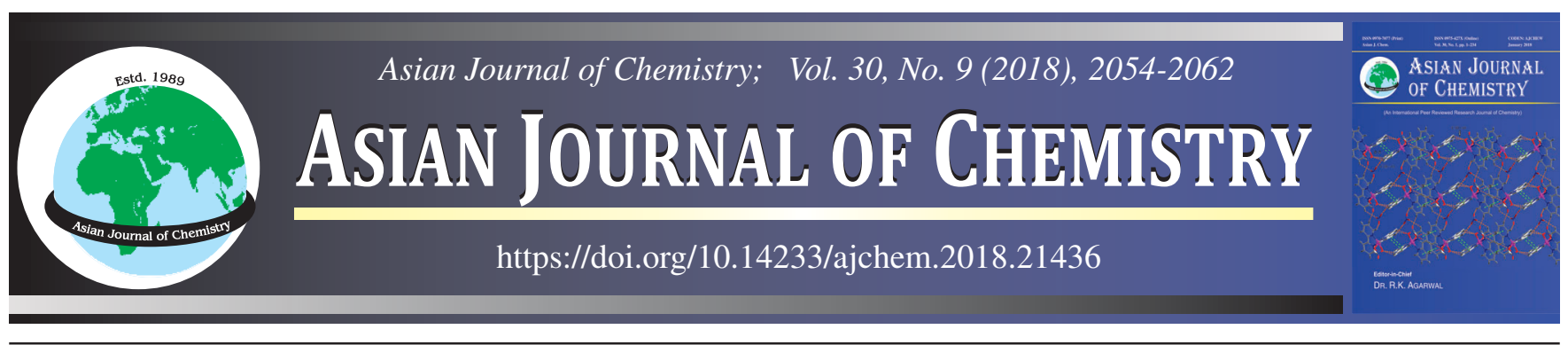

\title{
Intermolecular Interactions and Refractive Indices: Experimental Data and Prediction of Oxygenated Fuel Additives with Hydrocarbons
}

\author{
Rekha Devi ${ }^{1}$, Suman Gahlyan $^{1}$, Manju Rani $^{2}$ and Sanjeev Maken ${ }^{1, *}$
}

${ }^{1}$ Department of Chemistry, Deenbandhu Chhotu Ram University of Science and Technology, Murthal-131 039, India

${ }^{2}$ Department of Chemical Engineering, Deenbandhu Chhotu Ram University of Science and Technology, Murthal-131 039, India

*Corresponding author: Fax: +91 130 2484119; E-mail: sanjeevmakin@gmail.com

Oxygen containing compounds specifically alkanol and ether are being used as oxygenate fuel additives. The refractive indices $\left(\mathrm{n}_{\mathrm{D}}\right)$ of oxygenated fuel additive, aliphatic and aromatic hydrocarbon and their mixtures were measured at $298.15 \mathrm{~K}$ to $318.15 \mathrm{~K}$. The mixtures selected were diisopropyl ether (DIPE) + benzene, toluene, $n$-alkane $\left(\mathrm{C}_{6}-\mathrm{C}_{8}\right)$ or benzene, toluene $(1)+$ alkane $(2)$ and all possible combinations of alkanes at 298.15 K to 318.15 K. Various mixing rules like Gladstone-Dale, Arago-Biot, Heller, Weiner, Newton and Erying-John were applied for the theoretical estimation of refractive index and compared with experimental refractive indices of binary mixtures. Polynomial equation was applied to the measured data to calculate standard deviation. The $\Delta \mathrm{n}$ were further interpreted in terms of intermolecular interactions between the oxygenated fuel additive, aliphatic and aromatic hydrocarbons.

1

Keywords: Oxygenate, Refractive index, Diisopropyl ether, Hydrocarbons, Intermolecular interactions.

\section{INTRODUCTION}

Increase in global warming and extinction of fossil fuels is a great issue now a day. So the researchers have much interest in the alternative bio-fuels [1-3], which include biodiesel, bioalcohol, vegetable oil, propane and other biomass sources. Bio-fuels are used as oxygenated compounds in motor fuel [4]. The oxygenates are the oxygen containing compounds such as ethers, alkanol and ester used as fuel additives. The oxygen present in these oxygenates help in complete combustion of fuel. These are also good antiknocking agent with high octane number $[5,6]$. Now days due to carcinogenic nature of benzene it needs to be replaced [7,8] and toluene is a good candidate as it has similar property as benzene but much less carcinogenic [9]. In this sense, it is an interesting research object to study the thermophysical of the oxygenate with hydrocarbons.

The dependency of volume, acoustic or optical properties of multi-component mixtures on concentration or temperature is a useful indicator for reality of significant effects resulting due to intermolecular interactions. Thus, these mixing properties have significance in design and simulation process. In previous work [10-28], the thermophysical properties like excess volume, excess enthalpy, deviation in viscosity and ultrasonic speed of the binary systems of oxygenate with hydrocarbons have been studied. In this paper, the refractive indices $\left(\mathrm{n}_{\mathrm{D}}\right)$ of diisopropyl ether (DIPE) + benzene, toluene, $n$-alkane $\left(\mathrm{C}_{6}-\mathrm{C}_{8}\right)$ or benzene, toluene (1) + alkane (2) and all possible combinations of alkanes at 298.15 to $318.15 \mathrm{~K}$ are reported. Redlich-Kister equation have been used to correlate experimental values of refractive indices of binary mixtures. These deviations in $\mathrm{n}_{\mathrm{D}}$ have been used to understand the nature of interaction between unlike molecules. Various mixing rules were applied for the theoretical estimation of $n_{D}$ and these estimated values have been compared quantitatively with experimental $\mathrm{n}_{\mathrm{D}}$ of binary mixtures.

\section{EXPERIMENTAL}

Diisopropyl ether was purified by standard procedure [29] and other chemicals e.g., $n$-octane, $n$-heptane, $n$-hexane, toluene and benzene (Merck, > $99 \%$ ) were doubly distilled and kept in amber coloured bottles over molecular sieves (4A). For the measurement of density of the purified samples, Anton Paar DSA-5000 was used with an uncertainty of $\pm 10^{-3} \mathrm{~kg} \mathrm{~m}^{-3}$.

This is an open access journal, and articles are distributed under the terms of the Creative Commons Attribution-NonCommercial 4.0 International (CC BY-NC 4.0) License, which allows others to copy and redistribute the material in any medium or format, remix, transform, and build upon the material, as long as appropriate credit is given and the new creations are licensed under the identical terms. 
$\mathrm{n}_{\mathrm{D}}$ data were measured with a refractometer (Abbemat200 ) with temperature controlled within $\pm 0.01 \mathrm{~K}$ having accuracy up to $\pm 1 \times 10^{-4}$. The binary mixtures were made using a balance of precision $\pm 0.1 \mathrm{mg}$ (OHAUS, AR224CN).

The densities $(\rho)$ and $n_{D}$ for pure compounds and their binary systems are given in Tables 1 and 2, respectively.

\section{RESULTS AND DISCUSSION}

Deviation in refractive index $(\Delta \mathrm{n})$ have been calculated as:

$$
\Delta \mathrm{n}=\mathrm{n}_{\mathrm{D}}-\sum_{\mathrm{i}=1}^{2} \mathrm{x}_{\mathrm{i}} \mathrm{n}_{\mathrm{Di}}
$$

TABLE-1

PURITIES, MEASURED DENSITIES ( $\rho$ ) AND REFRACTIVE INDICES $\left(\mathrm{n}_{\mathrm{D}}\right.$ ) OF PURE COMPOUNDS

\begin{tabular}{|c|c|c|c|c|c|c|}
\hline \multirow{2}{*}{ Compound } & \multirow{2}{*}{$\begin{array}{l}\text { GC results } \\
\text { (wt. \%) }\end{array}$} & \multirow{2}{*}{ Temp. (K) } & \multicolumn{2}{|c|}{$\rho\left(\mathrm{kg} \mathrm{m}^{-3}\right)$} & \multicolumn{2}{|c|}{$\mathrm{n}_{\mathrm{D}}$} \\
\hline & & & This work & Literature & This work & Literature \\
\hline \multirow{3}{*}{ DIPE } & \multirow{3}{*}{99.7} & 298.15 & 718.522 & $718.207[30]$ & 1.3654 & $1.3655[31]$ \\
\hline & & 308.15 & 707.966 & $707.664[30]$ & 1.3598 & $1.3605[31]$ \\
\hline & & 318.15 & 697.220 & $696.931[30]$ & 1.3541 & \\
\hline \multirow{3}{*}{ Benzene } & \multirow{3}{*}{99.8} & 298.15 & 873.612 & $873.550[32]$ & 1.4972 & 1.4977 [32] \\
\hline & & 308.15 & 862.894 & 862.930 [32] & 1.4899 & 1.4910 [33] \\
\hline & & 318.15 & 852.103 & & 1.4821 & \\
\hline \multirow{3}{*}{ Toluene } & \multirow{3}{*}{99.8} & 298.15 & 862.058 & $862.220[32]$ & 1.4937 & $1.4944[32]$ \\
\hline & & 308.15 & 852.715 & $852.880[32]$ & 1.4877 & 1.4887 [32] \\
\hline & & 318.15 & 843.303 & & 1.4815 & \\
\hline \multirow{3}{*}{$n$-Hexane } & \multirow{3}{*}{99.6} & 298.15 & 655.296 & $654.800[34]$ & 1.3733 & $1.3736[34]$ \\
\hline & & 308.15 & 646.128 & $645.780[35]$ & 1.3679 & $1.3670[36]$ \\
\hline & & 318.15 & 636.779 & $636.390[35]$ & 1.3624 & \\
\hline \multirow{3}{*}{$n$-Heptane } & \multirow{3}{*}{99.7} & 298.15 & 679.575 & $679.750[37]$ & 1.3846 & $1.3850[34]$ \\
\hline & & 308.15 & 671.018 & 671.190 [37] & 1.3793 & 1.3803 [38] \\
\hline & & 318.15 & 662.341 & $662.500[37]$ & 1.3740 & \\
\hline \multirow{3}{*}{$n$-Octane } & \multirow{3}{*}{99.8} & 298.15 & 698.718 & $698.570[37]$ & 1.3953 & $1.3960[34]$ \\
\hline & & 308.15 & 690.596 & $690.440[37]$ & 1.3902 & 1.3908 [38] \\
\hline & & 318.15 & 682.380 & $682.230[37]$ & 1.3853 & \\
\hline
\end{tabular}

TABLE-2

REFRACTIVE INDEX $\left(\mathrm{n}_{\mathrm{D}}\right)$ AND DEVIATION IN REFRACTIVE INDEX $(\Delta \mathrm{n})$ FOR BINARY SYSTEMS AT DIFFERENT TEMPERATURES

\begin{tabular}{|c|c|c|c|c|c|c|}
\hline \multirow{2}{*}{$\mathrm{x}_{1}$} & \multicolumn{2}{|c|}{$298.15 \mathrm{~K}$} & \multicolumn{2}{|c|}{$308.15 \mathrm{~K}$} & \multicolumn{2}{|c|}{$318.15 \mathrm{~K}$} \\
\hline & $\mathrm{n}_{\mathrm{D}}$ & $\Delta \mathrm{n}$ & $\mathrm{n}_{\mathrm{D}}$ & $\Delta \mathrm{n}$ & $\mathrm{n}_{\mathrm{D}}$ & $\Delta \mathrm{n}$ \\
\hline \multicolumn{7}{|c|}{ DIPE (1) + Benzene (2) } \\
\hline 0.0491 & 1.4881 & -0.0026 & 1.4815 & -0.0020 & 1.4743 & -0.0015 \\
\hline 0.1058 & 1.4772 & -0.0061 & 1.4709 & -0.0052 & 1.4647 & -0.0039 \\
\hline 0.1745 & 1.4653 & -0.0089 & 1.4589 & -0.0083 & 1.4523 & -0.0075 \\
\hline 0.2149 & 1.4587 & -0.0102 & 1.4525 & -0.0094 & 1.4465 & -0.0081 \\
\hline 0.3064 & 1.4442 & -0.0126 & 1.4389 & -0.0111 & 1.4333 & -0.0096 \\
\hline 0.4302 & 1.4272 & -0.0133 & 1.4218 & -0.0121 & 1.4175 & -0.0095 \\
\hline 0.5168 & 1.4154 & -0.0137 & 1.4098 & -0.0129 & 1.4052 & -0.0108 \\
\hline 0.6222 & 1.4034 & -0.0118 & 1.3976 & -0.0114 & 1.3918 & -0.0107 \\
\hline 0.7258 & 1.3919 & -0.0097 & 1.3863 & -0.0092 & 1.381 & -0.0082 \\
\hline 0.8105 & 1.3832 & -0.0072 & 1.3777 & -0.0068 & 1.3743 & -0.0041 \\
\hline 0.9334 & 1.3708 & -0.0034 & 1.3657 & -0.0028 & 1.3603 & -0.0023 \\
\hline \multicolumn{7}{|c|}{ DIPE (1) + Toluene (2) } \\
\hline 0.0400 & 1.4877 & -0.0009 & 1.4819 & -0.0007 & 1.4762 & -0.0002 \\
\hline 0.0875 & 1.4808 & -0.0017 & 1.4754 & -0.0011 & 1.4701 & -0.0002 \\
\hline 0.1530 & 1.4705 & -0.0036 & 1.4658 & -0.0023 & 1.4605 & -0.0015 \\
\hline 0.2243 & 1.4599 & -0.0050 & 1.4556 & -0.0034 & 1.4501 & -0.0028 \\
\hline 0.3022 & 1.4485 & -0.0064 & 1.4446 & -0.0044 & 1.4377 & -0.0053 \\
\hline 0.3797 & 1.4385 & -0.0065 & 1.4327 & -0.0064 & 1.4268 & -0.0063 \\
\hline 0.4600 & 1.4279 & -0.0068 & 1.4219 & -0.0070 & 1.4159 & -0.0070 \\
\hline 0.5473 & 1.4168 & -0.0067 & 1.4113 & -0.0064 & 1.4053 & -0.0065 \\
\hline 0.6867 & 1.4001 & -0.0055 & 1.3968 & -0.0031 & 1.3905 & -0.0035 \\
\hline 0.7940 & 1.3878 & -0.0040 & 1.3834 & -0.0028 & 1.3786 & -0.0017 \\
\hline 0.9072 & 1.3755 & -0.0018 & 1.3709 & -0.0008 & 1.3649 & -0.0010 \\
\hline
\end{tabular}




\begin{tabular}{|c|c|c|c|c|c|c|}
\hline \multicolumn{7}{|c|}{ DIPE (1) $+n$-Hexane (2) } \\
\hline 0.0399 & 1.3722 & -0.0008 & 1.3669 & -0.0007 & 1.3614 & -0.0007 \\
\hline 0.1031 & 1.3714 & -0.0011 & 1.3663 & -0.0008 & 1.3608 & -0.0007 \\
\hline 0.1832 & 1.3707 & -0.0012 & 1.3656 & -0.0008 & 1.3601 & -0.0008 \\
\hline 0.2459 & 1.3701 & -0.0013 & 1.3648 & -0.0011 & 1.3591 & -0.0013 \\
\hline 0.3603 & 1.3692 & -0.0013 & 1.3637 & -0.0013 & 1.358 & -0.0014 \\
\hline 0.4834 & 1.3681 & -0.0014 & 1.3625 & -0.0015 & 1.3568 & -0.0016 \\
\hline 0.5624 & 1.3676 & -0.0013 & 1.3621 & -0.0012 & 1.3564 & -0.0013 \\
\hline 0.6891 & 1.367 & -0.0009 & 1.3615 & -0.0008 & 1.3556 & -0.0011 \\
\hline 0.7958 & 1.3668 & -0.0002 & 1.3609 & -0.0006 & 1.3551 & -0.0007 \\
\hline 0.9091 & 1.366 & -0.0001 & 1.3604 & -0.0001 & 1.3547 & -0.0002 \\
\hline 0.9577 & 1.3657 & 0.0000 & 1.3601 & 0.0000 & 1.3544 & -0.0001 \\
\hline \multicolumn{7}{|c|}{ DIPE (1) $+n$-Heptane (2) } \\
\hline 0.0398 & 1.3843 & 0.0005 & 1.3791 & 0.0006 & 1.3738 & 0.0006 \\
\hline 0.1061 & 1.3832 & 0.0006 & 1.3779 & 0.0007 & 1.3727 & 0.0008 \\
\hline 0.2002 & 1.3818 & 0.0010 & 1.3765 & 0.0011 & 1.3714 & 0.0014 \\
\hline 0.2830 & 1.3806 & 0.0014 & 1.3754 & 0.0016 & 1.3703 & 0.0019 \\
\hline 0.3965 & 1.3788 & 0.0018 & 1.3736 & 0.0020 & 1.3683 & 0.0022 \\
\hline 0.5073 & 1.3769 & 0.0020 & 1.3717 & 0.0023 & 1.3663 & 0.0024 \\
\hline 0.6051 & 1.3749 & 0.0019 & 1.3695 & 0.0020 & 1.3642 & 0.0022 \\
\hline 0.7157 & 1.3722 & 0.0013 & 1.3671 & 0.0018 & 1.3617 & 0.0019 \\
\hline 0.8200 & 1.3699 & 0.0010 & 1.3648 & 0.0015 & 1.3591 & 0.0014 \\
\hline 0.9189 & 1.3678 & 0.0008 & 1.3624 & 0.0010 & 1.357 & 0.0013 \\
\hline 0.9711 & 1.3664 & 0.0004 & 1.3608 & 0.0004 & 1.3553 & 0.0006 \\
\hline \multicolumn{7}{|c|}{ DIPE (1) $+n$-Octane (2) } \\
\hline 0.0475 & 1.3944 & 0.0005 & 1.3891 & 0.0003 & 1.3844 & 0.0006 \\
\hline 0.1172 & 1.3926 & 0.0008 & 1.3873 & 0.0007 & 1.3825 & 0.0009 \\
\hline 0.2036 & 1.3903 & 0.0011 & 1.3852 & 0.0012 & 1.3802 & 0.0013 \\
\hline 0.2917 & 1.3881 & 0.0015 & 1.3831 & 0.0018 & 1.3781 & 0.0019 \\
\hline 0.3958 & 1.3852 & 0.0017 & 1.3801 & 0.0019 & 1.3751 & 0.0021 \\
\hline 0.5047 & 1.3821 & 0.0019 & 1.3769 & 0.0020 & 1.3718 & 0.0022 \\
\hline 0.6067 & 1.3789 & 0.0017 & 1.3737 & 0.0019 & 1.3685 & 0.0021 \\
\hline 0.7233 & 1.3752 & 0.0015 & 1.3699 & 0.0017 & 1.3647 & 0.0020 \\
\hline 0.8296 & 1.3718 & 0.0013 & 1.3662 & 0.0012 & 1.3612 & 0.0018 \\
\hline 0.9241 & 1.3686 & 0.0009 & 1.3633 & 0.0012 & 1.3579 & 0.0014 \\
\hline 0.9619 & 1.3672 & 0.0007 & 1.3618 & 0.0008 & 1.3561 & 0.0008 \\
\hline \multicolumn{7}{|c|}{ Benzene (1) $+n$-Hexane (2) } \\
\hline 0.0578 & 1.3769 & -0.0037 & 1.3713 & -0.0039 & 1.3657 & -0.0038 \\
\hline 0.1429 & 1.3841 & -0.0071 & 1.378 & -0.0076 & 1.3722 & -0.0075 \\
\hline 0.2477 & 1.3936 & -0.0105 & 1.3874 & -0.0109 & 1.3812 & -0.0110 \\
\hline 0.3224 & 1.401 & -0.0124 & 1.3948 & -0.0126 & 1.389 & -0.0121 \\
\hline 0.4592 & 1.4158 & -0.0145 & 1.4103 & -0.0138 & 1.4059 & -0.0116 \\
\hline 0.5648 & 1.4283 & -0.0151 & 1.4216 & -0.0153 & 1.4158 & -0.0143 \\
\hline 0.6742 & 1.4437 & -0.0132 & 1.4373 & -0.0130 & 1.4316 & -0.0116 \\
\hline 0.7527 & 1.4546 & -0.0120 & 1.4479 & -0.0119 & 1.4419 & -0.0106 \\
\hline 0.8199 & 1.4642 & -0.0107 & 1.458 & -0.0100 & 1.452 & -0.0086 \\
\hline 0.8860 & 1.4752 & -0.0079 & 1.4687 & -0.0073 & 1.4629 & -0.0056 \\
\hline 0.9470 & 1.4929 & 0.0023 & 1.4864 & 0.0029 & 1.4796 & 0.0038 \\
\hline \multicolumn{7}{|c|}{ Benzene (1) $+n$-Heptane (2) } \\
\hline 0.0756 & 1.3896 & -0.0046 & 1.3877 & -0.0016 & 1.3822 & -0.0020 \\
\hline 0.1647 & 1.3962 & -0.0079 & 1.3919 & -0.0071 & 1.3873 & -0.0063 \\
\hline 0.2800 & 1.4047 & -0.0123 & 1.3978 & -0.0138 & 1.3919 & -0.0139 \\
\hline 0.3631 & 1.4099 & -0.0164 & 1.4026 & -0.0180 & 1.3951 & -0.0196 \\
\hline 0.4848 & 1.4223 & -0.0175 & 1.4156 & -0.0182 & 1.4089 & -0.0186 \\
\hline 0.6328 & 1.4382 & -0.0181 & 1.4317 & -0.0183 & 1.4249 & -0.0183 \\
\hline 0.7026 & 1.4471 & -0.0170 & 1.4415 & -0.0160 & 1.4337 & -0.0169 \\
\hline 0.7737 & 1.4572 & -0.0148 & 1.4478 & -0.0175 & 1.4416 & -0.0165 \\
\hline 0.8402 & 1.4648 & -0.0146 & 1.4579 & -0.0146 & 1.4501 & -0.0151 \\
\hline 0.8936 & 1.4736 & -0.0118 & 1.4672 & -0.0111 & 1.4597 & -0.0111 \\
\hline 0.9494 & 1.4886 & -0.0030 & 1.4808 & -0.0036 & 1.472 & -0.0047 \\
\hline
\end{tabular}




\begin{tabular}{|c|c|c|c|c|c|c|}
\hline \multicolumn{7}{|c|}{ Benzene $(1)+n$-Octane $(2)$} \\
\hline 0.0647 & 1.3982 & -0.004 & 1.3922 & -0.0046 & 1.3868 & -0.0050 \\
\hline 0.1477 & 1.4028 & -0.007 & 1.3966 & -0.0085 & 1.3914 & -0.0084 \\
\hline 0.2713 & 1.4097 & -0.013 & 1.4013 & -0.0161 & 1.3954 & -0.0163 \\
\hline 0.3634 & 1.4163 & -0.016 & 1.4096 & -0.0170 & 1.4035 & -0.0171 \\
\hline 0.4416 & 1.4226 & -0.018 & 1.4138 & -0.0205 & 1.4078 & -0.0204 \\
\hline 0.5695 & 1.4342 & -0.019 & 1.4276 & -0.0195 & 1.4179 & -0.0226 \\
\hline 0.6790 & 1.4458 & -0.019 & 1.4388 & -0.0192 & 1.429 & -0.0221 \\
\hline 0.7369 & 1.4533 & -0.017 & 1.4466 & -0.0171 & 1.4392 & -0.0175 \\
\hline 0.8240 & 1.4663 & -0.013 & 1.4585 & -0.0139 & 1.4508 & -0.0143 \\
\hline 0.9109 & 1.4794 & -0.009 & 1.4725 & -0.0085 & 1.4653 & -0.0082 \\
\hline 0.9567 & 1.4885 & -0.004 & 1.4812 & -0.0044 & 1.4732 & -0.0047 \\
\hline \multicolumn{7}{|c|}{ Toluene (1) $+n$-Hexane (2) } \\
\hline 0.0513 & 1.3778 & -0.0017 & 1.3731 & -0.0009 & 1.3674 & -0.0011 \\
\hline 0.1268 & 1.3853 & -0.0033 & 1.3806 & -0.0025 & 1.3761 & -0.0014 \\
\hline 0.2149 & 1.3942 & -0.0050 & 1.3889 & -0.0047 & 1.3844 & -0.0036 \\
\hline 0.2875 & 1.4021 & -0.0058 & 1.3969 & -0.0054 & 1.3924 & -0.0042 \\
\hline 0.4089 & 1.4161 & -0.0064 & 1.4107 & -0.0062 & 1.4057 & -0.0054 \\
\hline 0.5180 & 1.4289 & -0.0068 & 1.4235 & -0.0065 & 1.4182 & -0.0059 \\
\hline 0.6311 & 1.4432 & -0.0061 & 1.4375 & -0.0060 & 1.432 & -0.0056 \\
\hline 0.7134 & 1.4539 & -0.0053 & 1.4478 & -0.0056 & 1.4429 & -0.0045 \\
\hline 0.8078 & 1.4669 & -0.0037 & 1.4615 & -0.0032 & 1.4561 & -0.0025 \\
\hline 0.9093 & 1.4806 & -0.0022 & 1.4749 & -0.0019 & 1.4691 & -0.0016 \\
\hline 0.9520 & 1.4865 & -0.0014 & 1.4805 & -0.0015 & 1.4744 & -0.0014 \\
\hline \multicolumn{7}{|c|}{ Toluene $(1)+n$-Heptane (2) } \\
\hline 0.0557 & 1.3894 & -0.0013 & 1.3841 & -0.0012 & 1.3786 & -0.0014 \\
\hline 0.1150 & 1.394 & -0.0032 & 1.3887 & -0.0031 & 1.3833 & -0.0031 \\
\hline 0.2130 & 1.4021 & -0.0057 & 1.3966 & -0.0058 & 1.391 & -0.0059 \\
\hline 0.3099 & 1.4107 & -0.0077 & 1.4049 & -0.0080 & 1.3992 & -0.0081 \\
\hline 0.4294 & 1.4218 & -0.0096 & 1.416 & -0.0098 & 1.4105 & -0.0097 \\
\hline 0.5055 & 1.4293 & -0.0105 & 1.4238 & -0.0103 & 1.4183 & -0.0100 \\
\hline 0.6126 & 1.4419 & -0.0095 & 1.4362 & -0.0095 & 1.4306 & -0.0093 \\
\hline 0.7040 & 1.4523 & -0.0091 & 1.4467 & -0.0089 & 1.4407 & -0.0090 \\
\hline 0.8255 & 1.4676 & -0.0071 & 1.4616 & -0.0072 & 1.4556 & -0.0071 \\
\hline 0.9190 & 1.4812 & -0.0037 & 1.475 & -0.0039 & 1.4687 & -0.0041 \\
\hline 0.9556 & 1.4858 & -0.0031 & 1.4797 & -0.0032 & 1.4738 & -0.0029 \\
\hline \multicolumn{7}{|c|}{ Toluene $(1)+n$-Octane (2) } \\
\hline 0.0587 & 1.3999 & -0.0012 & 1.3951 & -0.0008 & 1.3902 & -0.0007 \\
\hline 0.1550 & 1.4046 & -0.0060 & 1.3996 & -0.0057 & 1.3946 & -0.0056 \\
\hline 0.2536 & 1.4121 & -0.0082 & 1.4069 & -0.0080 & 1.4015 & -0.0082 \\
\hline 0.3542 & 1.4196 & -0.0106 & 1.4141 & -0.0106 & 1.4084 & -0.0110 \\
\hline 0.4731 & 1.4298 & -0.0121 & 1.4243 & -0.0120 & 1.4186 & -0.0122 \\
\hline 0.5751 & 1.4394 & -0.0125 & 1.4337 & -0.0126 & 1.4279 & -0.0127 \\
\hline 0.6441 & 1.4467 & -0.0120 & 1.4411 & -0.0119 & 1.4349 & -0.0124 \\
\hline 0.7281 & 1.4561 & -0.0108 & 1.4503 & -0.0109 & 1.4439 & -0.0114 \\
\hline 0.8221 & 1.4679 & -0.0083 & 1.4618 & -0.0086 & 1.4558 & -0.0086 \\
\hline 0.8824 & 1.4753 & -0.0068 & 1.4699 & -0.0063 & 1.4639 & -0.0063 \\
\hline 0.9593 & 1.4884 & -0.0013 & 1.4819 & -0.0018 & 1.4758 & -0.0018 \\
\hline \multicolumn{7}{|c|}{$n$-Hexane $+n$-Heptane (2) } \\
\hline 0.0413 & 1.3851 & -0.0002 & 1.3804 & -0.0002 & 1.3754 & -0.0002 \\
\hline 0.1134 & 1.3842 & -0.0002 & 1.3794 & -0.0002 & 1.3744 & -0.0002 \\
\hline 0.1963 & 1.3831 & -0.0002 & 1.3783 & -0.0002 & 1.3732 & -0.0003 \\
\hline 0.2827 & 1.382 & -0.0003 & 1.3771 & -0.0003 & 1.372 & -0.0003 \\
\hline 0.4067 & 1.3804 & -0.0003 & 1.3754 & -0.0003 & 1.3702 & -0.0004 \\
\hline 0.5276 & 1.3789 & -0.0003 & 1.3738 & -0.0003 & 1.3685 & -0.0004 \\
\hline 0.6030 & 1.378 & -0.0003 & 1.3729 & -0.0002 & 1.3675 & -0.0004 \\
\hline 0.7246 & 1.3765 & -0.0002 & 1.3713 & -0.0002 & 1.3659 & -0.0003 \\
\hline 0.8268 & 1.3753 & -0.0002 & 1.37 & -0.0002 & 1.3645 & -0.0003 \\
\hline 0.8874 & 1.3745 & -0.0002 & 1.3692 & -0.0002 & 1.3639 & -0.0001 \\
\hline 0.9496 & 1.3738 & -0.0001 & 1.3684 & -0.0002 & 1.3631 & 0.0000 \\
\hline
\end{tabular}




\begin{tabular}{|c|c|c|c|c|c|c|}
\hline \multicolumn{7}{|c|}{$n$-Hexane $+n$-Octane (2) } \\
\hline 0.0513 & 1.3944 & 0.0003 & 1.3896 & 0.0004 & 1.3847 & 0.0004 \\
\hline 0.1233 & 1.3933 & 0.0008 & 1.3883 & 0.0007 & 1.3834 & 0.0007 \\
\hline 0.2153 & 1.3914 & 0.0009 & 1.3865 & 0.0009 & 1.3818 & 0.0013 \\
\hline 0.3073 & 1.3897 & 0.0012 & 1.3849 & 0.0014 & 1.3801 & 0.0017 \\
\hline 0.4324 & 1.3877 & 0.0020 & 1.3835 & 0.0028 & 1.3783 & 0.0028 \\
\hline 0.5524 & 1.3857 & 0.0026 & 1.3812 & 0.0032 & 1.3764 & 0.0037 \\
\hline 0.6357 & 1.3834 & 0.0021 & 1.3789 & 0.0028 & 1.3743 & 0.0035 \\
\hline 0.7478 & 1.3808 & 0.0020 & 1.3761 & 0.0025 & 1.3712 & 0.0030 \\
\hline 0.8384 & 1.3781 & 0.0013 & 1.3728 & 0.0013 & 1.3679 & 0.0018 \\
\hline 0.8975 & 1.3765 & 0.0010 & 1.3711 & 0.0009 & 1.3657 & 0.0009 \\
\hline 0.9498 & 1.3746 & 0.0002 & 1.3693 & 0.0003 & 1.3639 & 0.0003 \\
\hline \multicolumn{7}{|c|}{$n$-Heptane $+n$-Octane (2) } \\
\hline 0.0509 & 1.3949 & 0.0001 & 1.3899 & 0.0003 & 1.385 & 0.0003 \\
\hline 0.1208 & 1.3942 & 0.0002 & 1.3893 & 0.0004 & 1.3843 & 0.0004 \\
\hline 0.2109 & 1.3936 & 0.0006 & 1.3885 & 0.0006 & 1.3835 & 0.0006 \\
\hline 0.2993 & 1.3927 & 0.0006 & 1.3877 & 0.0008 & 1.3826 & 0.0007 \\
\hline 0.4122 & 1.3915 & 0.0006 & 1.3865 & 0.0008 & 1.3814 & 0.0008 \\
\hline 0.5249 & 1.3905 & 0.0008 & 1.3853 & 0.0008 & 1.3803 & 0.0009 \\
\hline 0.6104 & 1.3895 & 0.0007 & 1.3843 & 0.0008 & 1.3792 & 0.0008 \\
\hline 0.7427 & 1.388 & 0.0006 & 1.3828 & 0.0007 & 1.3776 & 0.0007 \\
\hline 0.8315 & 1.3869 & 0.0005 & 1.3818 & 0.0007 & 1.3765 & 0.0006 \\
\hline 0.9190 & 1.3859 & 0.0004 & 1.3807 & 0.0005 & 1.3753 & 0.0004 \\
\hline 0.9639 & 1.3851 & 0.0001 & 1.3799 & 0.0002 & 1.3746 & 0.0002 \\
\hline
\end{tabular}

where $x_{i}$ and $n_{D i}$ represent the mole fraction and the refractive index of the $\mathrm{i}^{\text {th }}$ component, respectively and $\mathrm{n}_{\mathrm{D}}$ is the refractive index of binary liquid mixture. Experimental $n_{D}$ and $\Delta \mathrm{n}$ data of binary systems are given in Table- 2 . The $\Delta \mathrm{n}$ values were also fitted to polynomial equation (Figs. 1 and 2).

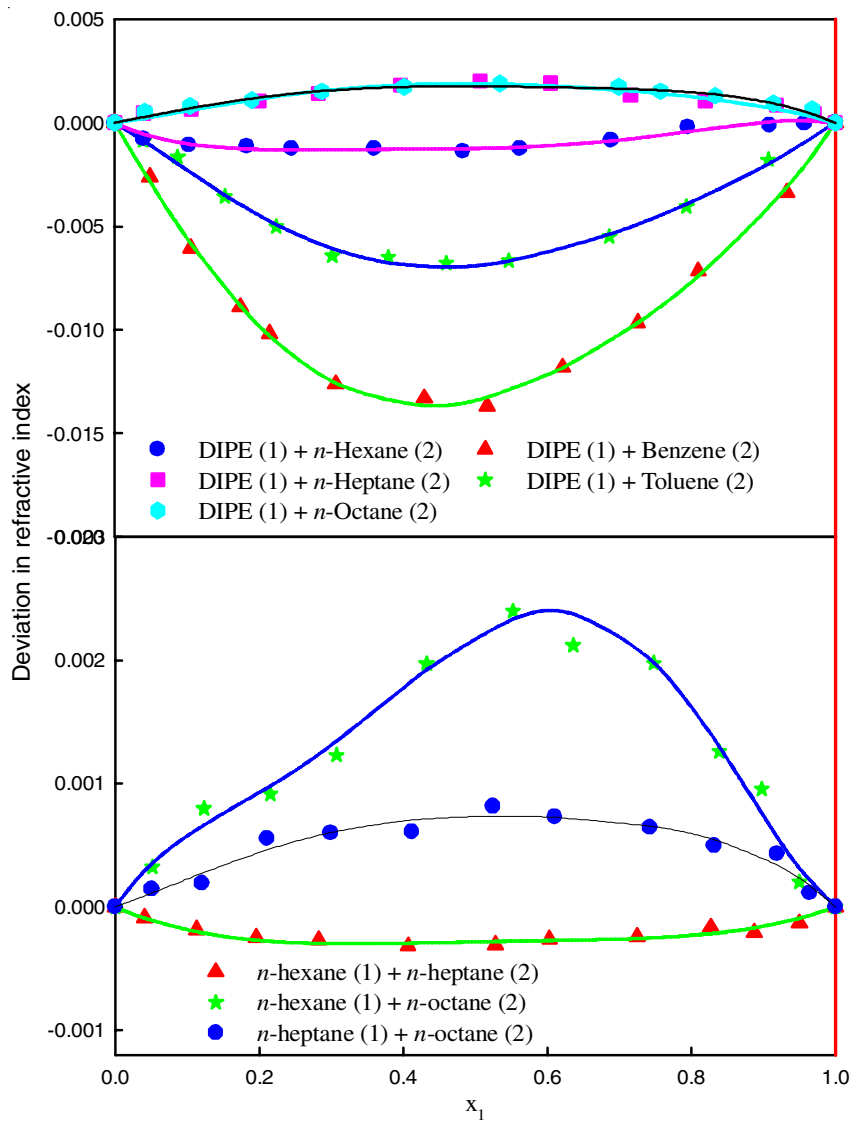

Fig. 1. Deviation in refractive index for the binary $(1+2)$ mixtures as a function of mole fraction of $\mathrm{x}_{1}$ at $298.15 \mathrm{~K}$. Symbols represent experimental values and lines represent values calculated from Redlich-Kister equation

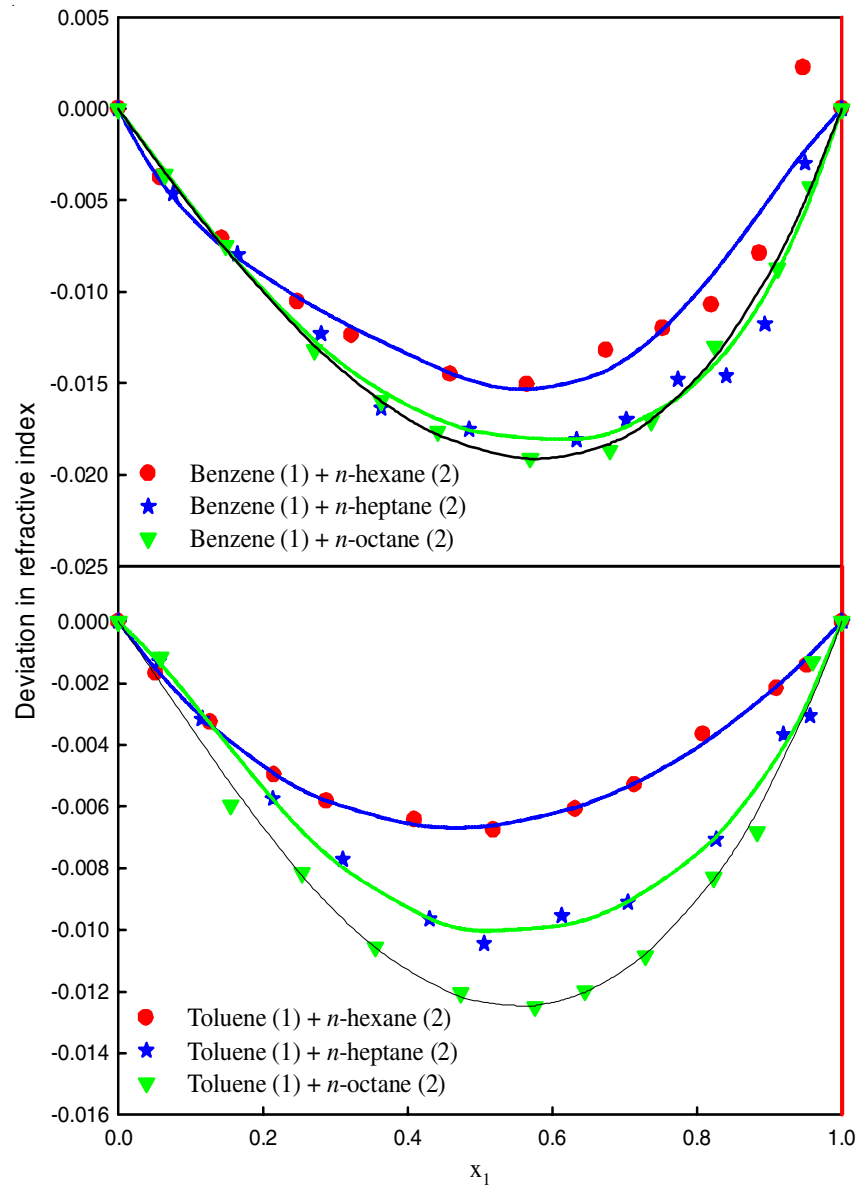

Fig. 2. Deviation in refractive index for the binary $(1+2)$ mixtures as a function of mole fraction of $x_{1}$ at $298.15 \mathrm{~K}$. Symbols represent experimental values and lines represent values calculated from Redlich-Kister equation

$$
\Delta \mathrm{n}=\mathrm{x}_{1}\left(1-\mathrm{x}_{\mathrm{i}}\right)\left(\sum_{\mathrm{n}=1}^{4} \mathrm{~A}^{(\mathrm{j})}\left(2 \mathrm{x}_{1}-1\right)^{(\mathrm{j}-1)}\right)
$$


where $\mathrm{A}^{(\mathrm{j})}$ are the adjustable parameters and calculated by fitting $\Delta \mathrm{n}$ data in eqn. 2 respective standard deviations $\sigma(\Delta \mathrm{n})$ were recorded in Table-3.
For quantitative determination of $n_{D}$ following mixing relations were used:

Arago-Biot (A-B):

TABLE-3

ADJUSTABLE PARAMETERS OF REDLICH-KISTER EQUATION $\left(\mathrm{A}^{(\mathrm{j})}\right)$ AND STANDARD DEVIATION $(\sigma)$

\begin{tabular}{|c|c|c|c|c|c|}
\hline Temperature $(\mathrm{K})$ & $\mathrm{A}^{(1)}$ & $\mathrm{A}^{(2)}$ & $\mathrm{A}^{(3)}$ & $\mathrm{A}^{(4)}$ & $\sigma$ \\
\hline \multicolumn{6}{|c|}{ DIPE (1) + Benzene (2) } \\
\hline 298.15 & -0.0536 & 0.0148 & -0.0029 & -0.0111 & 0.0000 \\
\hline 308.15 & -0.0502 & 0.0089 & 0.0010 & -0.0021 & 0.0004 \\
\hline 318.15 & -0.0432 & 0.0045 & 0.0079 & 0.0111 & 0.0010 \\
\hline \multicolumn{6}{|c|}{ DIPE (1) + Toluene (2) } \\
\hline 298.15 & -0.0276 & 0.0063 & 0.0047 & -0.0083 & 0.0002 \\
\hline 308.15 & -0.0253 & 0.0087 & 0.0236 & -0.0092 & 0.0006 \\
\hline 318.15 & -0.0263 & 0.0129 & 0.0329 & -0.0238 & 0.0004 \\
\hline \multicolumn{6}{|c|}{ DIPE (1) $+n$-Hexane (2) } \\
\hline 298.15 & -0.0050 & 0.0013 & -0.0010 & 0.0092 & 0.0001 \\
\hline 308.15 & -0.0052 & 0.0008 & 0.0009 & 0.0053 & 0.0002 \\
\hline 318.15 & -0.0059 & 0.0003 & 0.0012 & 0.0055 & 0.0002 \\
\hline \multicolumn{6}{|c|}{ DIPE (1) $+n$-Heptane (2) } \\
\hline 298.15 & 0.0076 & -0.0005 & -0.0001 & 0.0031 & 0.0002 \\
\hline 308.15 & 0.0084 & 0.0004 & 0.0016 & 0.0038 & 0.0002 \\
\hline 318.15 & 0.0091 & -0.0009 & 0.0032 & 0.0061 & 0.0002 \\
\hline \multicolumn{6}{|c|}{ DIPE (1) $+n$-Octane (2) } \\
\hline 298.15 & 0.0071 & -0.0002 & 0.0038 & 0.0043 & 0.0001 \\
\hline 308.15 & 0.0079 & -0.0021 & 0.0026 & 0.0105 & 0.0002 \\
\hline 318.15 & 0.0086 & -0.0006 & 0.0057 & 0.0094 & 0.0002 \\
\hline \multicolumn{6}{|c|}{ Benzene (1) $+n$-Hexane (2) } \\
\hline 298.15 & -0.0598 & -0.0204 & -0.0007 & 0.0428 & 0.0014 \\
\hline 308.15 & -0.0595 & -0.0209 & -0.0004 & 0.0568 & 0.0022 \\
\hline 318.15 & -0.0544 & -0.0185 & 0.0004 & 0.0694 & 0.0023 \\
\hline \multicolumn{6}{|c|}{ Benzene (1) $+n$-Heptane (2) } \\
\hline 298.15 & -0.0708 & -0.0195 & -0.0193 & -0.0184 & 0.0002 \\
\hline 308.15 & -0.0764 & -0.0048 & 0.0000 & -0.0702 & 0.0014 \\
\hline 318.15 & -0.0788 & 0.0019 & 0.0047 & -0.0891 & 0.0012 \\
\hline \multicolumn{6}{|c|}{ Benzene (1) $+n$-Octane (2) } \\
\hline 298.15 & -0.0748 & -0.0226 & -0.0074 & -0.0049 & 0.0000 \\
\hline 308.15 & -0.0804 & -0.0095 & -0.0105 & -0.0165 & 0.0008 \\
\hline 318.15 & -0.0865 & -0.0281 & 0.0000 & 0.0183 & 0.0011 \\
\hline \multicolumn{6}{|c|}{ Toluene (1) $+n$-Hexane (2) } \\
\hline 298.15 & -0.0266 & 0.0032 & -0.0024 & 0.0001 & 0.0002 \\
\hline 308.15 & -0.0263 & 0.0027 & 0.0040 & -0.0044 & 0.0004 \\
\hline 318.15 & -0.0233 & 0.0002 & 0.0100 & -0.0033 & 0.0004 \\
\hline \multicolumn{6}{|c|}{ Toluene (1) $+n$-Heptane (2) } \\
\hline 298.15 & -0.0401 & -0.0054 & -0.0012 & -0.0159 & 0.0004 \\
\hline 308.15 & -0.0401 & -0.0020 & -0.0023 & -0.0246 & 0.0003 \\
\hline 318.15 & -0.0394 & -0.0015 & -0.0048 & -0.0242 & 0.0002 \\
\hline \multicolumn{6}{|c|}{ Toluene (1) +n-Octane (2) } \\
\hline 298.15 & -0.0494 & -0.0104 & 0.0006 & -0.0050 & 0.0006 \\
\hline 308.15 & -0.0497 & -0.0094 & 0.0032 & -0.0108 & 0.0005 \\
\hline 318.15 & -0.0511 & -0.0109 & 0.0056 & -0.0090 & 0.0006 \\
\hline \multicolumn{6}{|c|}{$n$-Hexane (1) $+n$-Heptane (2) } \\
\hline 298.15 & $-1.12 \times 10^{-3}$ & $2.23 \times 10^{-4}$ & $-1.22 \times 10^{-3}$ & $-4.60 \times 10^{-5}$ & $4.90 \times 10^{-5}$ \\
\hline 308.15 & $-1.15 \times 10^{-3}$ & $3.60 \times 10^{-4}$ & $-1.09 \times 10^{-3}$ & $-5.47 \times 10^{-4}$ & $5.31 \times 10^{-5}$ \\
\hline 318.15 & $4.24 \times 10^{-4}$ & $1.24 \times 10^{-3}$ & $-3.31 \times 10^{-3}$ & $9.01 \times 10^{-4}$ & $3.27 \times 10^{-4}$ \\
\hline \multicolumn{6}{|c|}{$n$-Hexane (1) $+n$-Octane (2) } \\
\hline 298.15 & $8.765 \times 10^{-3}$ & $6.783 \times 10^{-3}$ & $-2.216 \times 10^{-3}$ & $-8.802 \times 10^{-3}$ & $1.791 \times 10^{-4}$ \\
\hline 308.15 & $1.170 \times 10^{-2}$ & $8.881 \times 10^{-3}$ & $-8.077 \times 10^{-3}$ & $-1.120 \times 10^{-2}$ & $2.134 \times 10^{-4}$ \\
\hline 318.15 & $1.310 \times 10^{-2}$ & $1.210 \times 10^{-2}$ & $-6.856 \times 10^{-3}$ & $-1.620 \times 10^{-2}$ & $8.508 \times 10^{-5}$ \\
\hline \multicolumn{6}{|c|}{$n$-Heptane (1) $+n$-Octane (2) } \\
\hline 298.15 & $2.95 \times 10^{-3}$ & $2.69 \times 10^{-4}$ & $8.21 \times 10^{-4}$ & $1.43 \times 10^{-3}$ & $8.12 \times 10^{-5}$ \\
\hline 308.15 & $3.14 \times 10^{-3}$ & $-4.39 \times 10^{-4}$ & $2.74 \times 10^{-3}$ & $2.42 \times 10^{-3}$ & $4.50 \times 10^{-5}$ \\
\hline 318.15 & $3.31 \times 10^{-3}$ & $5.00 \times 10^{-4}$ & $1.43 \times 10^{-3}$ & $-4.00 \times 10^{-5}$ & $5.83 \times 10^{-5}$ \\
\hline
\end{tabular}




$$
\mathrm{n}_{\mathrm{D}}=\mathrm{n}_{\mathrm{D} 1} \phi_{1}+\mathrm{n}_{\mathrm{D} 2} \phi_{2}
$$

Gladstone-Dale (G-D):

$$
\mathrm{n}_{\mathrm{D}}-1=\left(\mathrm{n}_{\mathrm{D} 1}-1\right) \phi_{1}+\left(\mathrm{n}_{\mathrm{D} 2}-1\right) \phi_{2}
$$

Lorentz-Lorenz (L-L):

$$
\frac{\mathrm{n}_{\mathrm{D}}^{2}-1}{\mathrm{n}_{\mathrm{D}}^{2}+2}=\left(\frac{\mathrm{n}_{\mathrm{D} 1}^{2}-1}{\mathrm{n}_{\mathrm{D}}^{2}+2}\right) \phi_{1}+\left(\frac{\mathrm{n}_{\mathrm{D} 2}^{2}-1}{\mathrm{n}_{\mathrm{D}}^{2}+2}\right) \phi_{2}
$$

Heller $(\mathrm{H})$ :

$$
\frac{\mathrm{n}_{\mathrm{D}}-1}{\mathrm{n}_{\mathrm{D}}}=\frac{3}{2}\left(\frac{\left(\mathrm{n}_{\mathrm{D} 2} / \mathrm{n}_{\mathrm{D} 1}\right)^{2}-1}{\left(\mathrm{n}_{\mathrm{D} 2} / \mathrm{n}_{\mathrm{D} 1}\right)^{2}+2}\right) \phi_{2}
$$

Weiner (W):

$$
\frac{\mathrm{n}_{\mathrm{D}}^{2}-\mathrm{n}_{\mathrm{D} 1}^{2}}{\mathrm{n}_{\mathrm{D}}^{2}+2 \mathrm{n}_{\mathrm{D} 2}^{2}}=\left(\frac{\mathrm{n}_{\mathrm{D} 2}^{2}-\mathrm{n}_{\mathrm{D} 1}^{2}}{\mathrm{n}_{\mathrm{D} 2}^{2}+2 \mathrm{n}_{\mathrm{D} 2}^{2}}\right) \phi_{2}
$$

Newton (Nw):

$$
\mathrm{n}_{\mathrm{D}}^{2}-1=\left(\mathrm{n}_{\mathrm{D} 1}^{2}-1\right) \phi_{1}+\left(\mathrm{n}_{\mathrm{D} 2}^{2}-1\right) \phi_{2}
$$

Eyring and John (E-J):

$$
\mathrm{n}_{\mathrm{D}}=\mathrm{n}_{\mathrm{D} 1} \phi_{1}^{2}+2\left(\mathrm{n}_{\mathrm{D} 1} \mathrm{n}_{\mathrm{D} 2}\right)^{1 / 2} \phi_{1} \phi_{2}+\mathrm{n}_{\mathrm{D} 2} \phi_{2}^{2}
$$

In all above equations, $n_{D}, n_{D 1}$ and $n_{D 2}$ are the refractive index of mixture and its pure component, respectively. $\phi_{1}$ and $\phi_{2}$ represent the volume fraction of pure components and given by

$$
\phi_{1}=\frac{\mathrm{x}_{1} \mathrm{~V}_{1}}{\sum \mathrm{x}_{\mathrm{i}} \mathrm{V}_{\mathrm{i}}} \text { and } \phi_{2}=\frac{\mathrm{x}_{2} \mathrm{~V}_{2}}{\sum \mathrm{x}_{\mathrm{i}} \mathrm{V}_{\mathrm{i}}}
$$

\begin{tabular}{|c|c|c|c|c|c|c|c|c|}
\hline Binary system & Temp. (K) & $\mathrm{AB}$ & GD & LL & Weiner & Heller & Newton & Eyring \\
\hline \multirow{3}{*}{ DIPE (1) + Benzene (2) } & 298.15 & 0.0260 & 0.0260 & 0.0247 & 0.0391 & 0.0243 & 0.0273 & 0.0254 \\
\hline & 308.15 & 0.0010 & 0.0010 & 0.0010 & 0.0011 & 0.0010 & 0.0010 & 0.0010 \\
\hline & 318.15 & 0.0041 & 0.0041 & 0.0053 & 0.0100 & 0.0055 & 0.0028 & 0.0047 \\
\hline \multirow{3}{*}{ DIPE (1) + Toluene (2) } & 298.15 & 0.0816 & 0.0816 & 0.0816 & 0.0807 & 0.0817 & 0.0815 & 0.0816 \\
\hline & 308.15 & 0.0035 & 0.0035 & 0.0048 & 0.0110 & 0.0051 & 0.0023 & 0.0042 \\
\hline & 318.15 & 0.0039 & 0.0039 & 0.0052 & 0.0106 & 0.0055 & 0.0028 & 0.6551 \\
\hline \multirow{3}{*}{ DIPE (1) $+n$-Hexane (2) } & 298.15 & 0.0020 & 0.0020 & 0.0020 & 0.0020 & 0.0020 & 0.0020 & 0.0020 \\
\hline & 308.15 & 0.0017 & 0.0017 & 0.0017 & 0.0015 & 0.0017 & 0.0017 & 0.0017 \\
\hline & 318.15 & 0.0011 & 0.0011 & 0.0011 & 0.0012 & 0.0011 & 0.0011 & 0.0011 \\
\hline \multirow{3}{*}{ DIPE (1) $+n$-Heptane (2) } & 298.15 & 0.0013 & 0.0013 & 0.0013 & 0.0016 & 0.0013 & 0.0013 & 0.0013 \\
\hline & 308.15 & 0.0009 & 0.0009 & 0.0010 & 0.0008 & 0.0010 & 0.0009 & 0.0010 \\
\hline & 318.15 & 0.0019 & 0.0019 & 0.0020 & 0.0017 & 0.0020 & 0.0019 & 0.0019 \\
\hline \multirow{3}{*}{ DIPE (1) $+n$-Octane (2) } & 298.15 & 0.0018 & 0.0018 & 0.0018 & 0.0024 & 0.0018 & 0.0019 & 0.0018 \\
\hline & 308.15 & 0.0010 & 0.0010 & 0.0010 & 0.0011 & 0.0010 & 0.0010 & 0.0010 \\
\hline & 318.15 & 0.0012 & 0.0012 & 0.0013 & 0.0009 & 0.0013 & 0.0011 & 0.0012 \\
\hline \multirow{3}{*}{ Benzene (1) $+n$-Hexane (2) } & 298.15 & 0.0037 & 0.0037 & 0.0029 & 0.0138 & 0.0031 & 0.0047 & 0.0033 \\
\hline & 308.15 & 0.0037 & 0.0037 & 0.0030 & 0.0136 & 0.0031 & 0.0046 & 0.0033 \\
\hline & 318.15 & 0.0034 & 0.0034 & 0.0031 & 0.0127 & 0.0030 & 0.0040 & 0.0032 \\
\hline \multirow{3}{*}{ Benzene (1) $+n$-Heptane (2) } & 298.15 & 0.0127 & 0.0127 & 0.0117 & 0.0192 & 0.0121 & 0.0137 & 0.0122 \\
\hline & 308.15 & 0.0057 & 0.0057 & 0.0048 & 0.0127 & 0.0052 & 0.0066 & 0.0053 \\
\hline & 318.15 & 0.0064 & 0.0064 & 0.0055 & 0.0130 & 0.0059 & 0.0072 & 0.0060 \\
\hline \multirow{3}{*}{ Benzene (1) $+n$-Octane (2) } & 298.15 & 0.0092 & 0.0092 & 0.0084 & 0.0153 & 0.0087 & 0.0100 & 0.0088 \\
\hline & 308.15 & 0.0052 & 0.0052 & 0.0045 & 0.0120 & 0.0046 & 0.0059 & 0.0048 \\
\hline & 318.15 & 0.0066 & 0.0066 & 0.0059 & 0.0129 & 0.0060 & 0.0074 & 0.0063 \\
\hline \multirow{3}{*}{ Toluene (1) $+n$-Hexane (2) } & 298.15 & 0.0061 & 0.0061 & 0.0072 & 0.0087 & 0.0070 & 0.0050 & 0.0067 \\
\hline & 308.15 & 0.0005 & 0.0005 & 0.0012 & 0.0107 & 0.0012 & 0.0013 & 0.0007 \\
\hline & 318.15 & 0.0010 & 0.0010 & 0.0020 & 0.0100 & 0.0020 & 0.0007 & 0.0015 \\
\hline \multirow{3}{*}{ Toluene (1) $+n$-Heptane (2) } & 298.15 & 0.0017 & 0.0017 & 0.0026 & 0.0082 & 0.0024 & 0.0009 & 0.0021 \\
\hline & 308.15 & 0.0014 & 0.0014 & 0.0007 & 0.0096 & 0.0013 & 0.0022 & 0.0010 \\
\hline & 318.15 & 0.0013 & 0.0013 & 0.0007 & 0.0095 & 0.0012 & 0.0022 & 0.0010 \\
\hline \multirow{3}{*}{ Toluene (1) + n-Octane (2) } & 298.15 & 0.0009 & 0.0009 & 0.0007 & 0.0076 & 0.0010 & 0.0015 & 0.0007 \\
\hline & 308.15 & 0.0020 & 0.0020 & 0.0013 & 0.0083 & 0.0016 & 0.0027 & 0.0016 \\
\hline & 318.15 & 0.0023 & 0.0023 & 0.0016 & 0.0084 & 0.0020 & 0.0030 & 0.0020 \\
\hline \multirow{3}{*}{$n$-Hexane $(1)+n$-Heptane (2) } & 298.15 & 0.0012 & 0.0012 & 0.0012 & 0.0013 & 0.0012 & 0.0012 & 0.0012 \\
\hline & 308.15 & 0.0010 & 0.0010 & 0.0010 & 0.0011 & 0.0010 & 0.0010 & 0.0010 \\
\hline & 318.15 & 0.0011 & 0.0011 & 0.0011 & 0.0012 & 0.0011 & 0.0011 & 0.0011 \\
\hline \multirow{3}{*}{$n$-Hexane $(1)+n$-Octane $(2)$} & 298.15 & 0.0012 & 0.0012 & 0.0012 & 0.0010 & 0.0012 & 0.0012 & 0.0012 \\
\hline & 308.15 & 0.0014 & 0.0014 & 0.0014 & 0.0012 & 0.0014 & 0.0013 & 0.0014 \\
\hline & 318.15 & 0.0017 & 0.0017 & 0.0017 & 0.0015 & 0.0017 & 0.0017 & 0.0017 \\
\hline \multirow{3}{*}{$n$-Heptane $(1)+n$-Octane $(2)$} & 298.15 & 0.0010 & 0.0010 & 0.0010 & 0.0010 & 0.0010 & 0.0010 & 0.0010 \\
\hline & 308.15 & 0.0005 & 0.0005 & 0.0006 & 0.0005 & 0.0006 & 0.0005 & 0.0006 \\
\hline & 318.15 & 0.0005 & 0.0005 & 0.0005 & 0.0005 & 0.0005 & 0.0005 & 0.0005 \\
\hline
\end{tabular}

From these mixing rules standard deviation were also calculated which are reported in Table-4. From Table-4 it can be seen that for all the binary mixtures all mixing rules have good results.

TABLE-4

STANDARD DEVIATION OF CALCULATED VALUES OF REFRACTIVE INDEX USING VARIOUS CORRELATIONS 
Figs. 1 and 2 show deviation in refractive index data versus $\mathrm{x}_{1}$ for these systems. The $\Delta \mathrm{n}$ values for the DIPE (1)+ $n$-heptane, $n$-octane (2) or $n$-hexane and $n$-heptane (1) $+n$ octane (2) systems are positive while negative for DIPE (1) + benzene, toluene or $n$-hexane (2), benzene or toluene (1) + $\mathrm{C}_{6}-\mathrm{C}_{8}$ (2) and $n$-hexane (1) $+n$-heptane (2) systems. The $\Delta \mathrm{n}$ values for DIPE (1) + hydrocarbon (2) at equimolar mixtures follow the sequence: $n$-octane $\approx n$-heptane $>n$-hexane $>$ toluene $>$ benzene while for benzene or toluene $(1)+$ hydrocarbon (2) the order is $n$-hexane $>n$-heptane $>n$-octane and for $n$-hexane (1) + hydrocarbon (2) the value is positive for $n$-octane and negative for $n$-heptane system.

As the refractive index is speed of light dependent property. With increase in density due to $n-\pi$ intermolecular interactions between aromatic hydrocarbon and lone pair of oxygen atom in DIPE the $n_{D}$ value also increase. The $\Delta \mathrm{n}$ in the DIPE + aromatic hydrocarbon mixtures for toluene are larger than benzene due to presence of one methyl group which increase the electron density on benzene ring for stronger $n-\pi$ interactions [39]. While in case of aliphatic $n$-heptane or $n$-octane there is absence of $\pi$-electrons only disorder in proper orientation of hydrocarbon occur, so for DIPE (1) + alkane (2) mixtures, medium gets rarer and refractive index decreases [40]. The deviation in refractive index is also influenced with temperature. The $\Delta \mathrm{n}$ for DIPE (1) + heptane or benzene (2) mixtures is plotted against composition in Fig. 3. As shown in Fig. 3 for DIPE (1) + heptane or benzene (2) systems, with

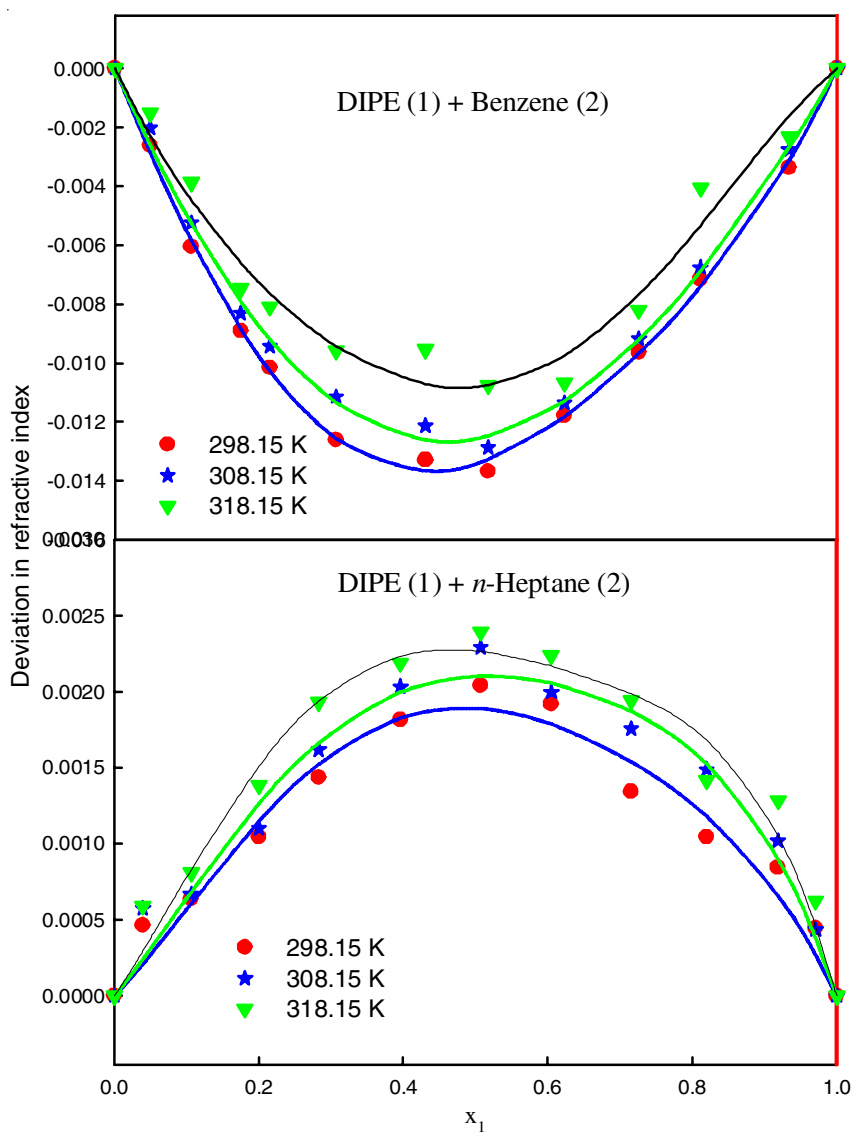

Fig. 3. Deviation in refractive index for the binary $(1+2)$ mixtures as a function of mole fraction of $\mathrm{x}_{1}$ at different temperature. Symbols represent experimental values and lines represent values calculated from Redlich-Kister equation increase in temperature $\Delta \mathrm{n}$ is also increasing in both positive as well as negative deviation.

\section{Conclusion}

Deviations in refractive index $(\Delta \mathrm{n})$ for DIPE and hydrocarbon systems were determined at 298.15 to $318.15 \mathrm{~K}$. The $\Delta \mathrm{n}$ values for the DIPE (1) $+n$-heptane, $n$-octane (2) or $n$ hexane and $n$-heptane (1) $+n$-octane (2) systems are positive while negative for DIPE (1) + benzene, toluene or $n$-hexane (2), benzene or toluene (1) $+\mathrm{C}_{6}-\mathrm{C}_{8}$ (2) and $n$-hexane (1) $+n$ heptane (2) systems. Various mixing rules applied for the theoretical estimation of $n_{D}$ have good results with experimental data for all binary systems.

\section{ACKNOWLEDGEMENTS}

One of the authors, Rekha Devi acknowledges CSIR, India for financial assistance as Senior Research Fellow.

\section{CONFLICT OF INTEREST}

The authors declare that there is no conflict of interests regarding the publication of this article.

\section{REFERENCES}

1. F.E.M. Alaoui, E.A. Montero, J.-P. Bazile, F. Aguilar and C. Boned, J. Chem. Thermodyn., 43, 1768 (2011); https://doi.org/10.1016/j.jct.2011.06.006.

2. M. Hajbabaei, G. Karavalakis, K.C. Johnson, J. Guthrie, A. Mitchell and T.D. Durbin, Fuel Process. Technol., 126, 402 (2014); https://doi.org/10.1016/j.fuproc.2014.04.030.

3. K.H. Kim, I.S. Choi, H.M. Kim, S.G. Wi and H.J. Bae, Bioresour. Technol., 153, 47 (2014);

https://doi.org/10.1016/j.biortech.2013.11.059

4. V.R. Surisetty, A.K. Dalai and J. Kozinski, Appl. Catal. A, 404, 1 (2011).

5. F. Ancillotti and V. Fattore, Fuel Process. Technol., 57, 163 (1998); https://doi.org/10.1016/S0378-3820(98)00081-2.

6. M. Karabektas, G. Ergen and M. Hosoz, Fuel, 115, 855 (2014); https://doi.org/10.1016/j.fuel.2012.12.062.

7. Regulation 715/2007 of the European Parliament Ad of the Council on Type Approval of Motor Vehicles with respect to Emissions from Light Passenger and Commercial Vehicles (Euro 5 and Euro 6) (2007).

8. Directive 2009/30/EC of the European Parliament Ad of the Council on the Specification of Petrol, Diesel and Gas-Oil and Introducing a Mechanism to Monitor and Reduce Greenhouse Gas Emissions (2009).

9. C. Dees, M. Askari and D. Henley, Environ. Health Perspect., 104 (Suppl. 6), 1289 (1996); https://doi.org/10.1289/ehp.961041289.

10. S. Verma, S. Gahlyan, M. Rani and S. Maken, Arab. J. Sci. Eng., (2018); https://doi.org/10.1007/s13369-018-3276-1.

11. N.T.A. Othman and M.P. Ngaliman, Indian J. Sci. Technol., 9, 1 (2016); https://doi.org/10.17485/ijst/2016/v9i21/95246.

12. K. Partibha, K. Kumar, S. Gahlyan, M. Rani and V. Bhankar, J. Mol. Liq., 259, 167 (2018); https://doi.org/10.1016/j.molliq.2018.03.025.

13. S. Gahlyan, S. Verma, M. Rani and S. Maken, Asian J. Chem., 30, 731 (2018); https://doi.org/10.14233/ajchem.2018.20685.

14. S. Gahlyan, S. Verma, M. Rani and S. Maken, Korean J. Chem. Eng., 35, 1167 (2018); https://doi.org/10.1007/s11814-018-0020-1.

15. S. Gahlyan, S. Verma, M. Rani and S. Maken, J. Mol. Liq., 258, 142 (2018); https://doi.org/10.1016/j.molliq.2018.03.003.

16. M. Rani, S. Gahlyan, H. Om, N. Verma and S. Maken, J. Mol. Liq., 194, 100 (2014); https://doi.org/10.1016/j.molliq.2014.01.016.

17. M. Rani and S. Maken, Thermochim. Acta, 559, 98 (2013); https://doi.org/10.1016/j.tca.2013.02.010. 
18. S. Gahlyan, M. Rani, I. Lee, I. Moon and S. Maken, Korean J. Chem. Eng., 32, 168 (2015); https://doi.org/10.1007/s11814-014-0200-6.

19. S. Gahlyan, M. Rani, S. Maken, H. Kwon, K. Tak and I. Moon, J. Ind. Eng. Chem., 23, 299 (2015); https://doi.org/10.1016/j.jiec.2014.08.032.

20. M. Rani and S. Maken, J. Ind. Eng. Chem., 18, 1694 (2012); https://doi.org/10.1016/j.jiec.2012.03.011.

21. M. Rani, S. Agarwal, P. Lahot and S. Maken, J. Ind. Eng. Chem., 19, 1715 (2013); https://doi.org/10.1016/i.jiec.2013.02.011.

22. S. Gahlyan, S. Verma, M. Rani and S. Maken, J. Mol. Liq., 244, 233 (2017); https://doi.org/10.1016/j.molliq.2017.09.015.

23. S. Gahlyan, S. Verma, M. Rani and S. Maken, Korean Chem. Eng. Res., 55, 668 (2017); https://doi.org/10.9713/kcer.2017.55.5.668.

24. S. Gahlyan, S. Verma, M. Rani and S. Maken, Korean Chem. Eng. Res., 55, 520 (2017); https://doi.org/10.9713/kcer.2017.55.4.520.

25. M. Rani and S. Maken, J. Ind. Eng. Chem., 19, 1760 (2013); https://doi.org/10.1016/j.jiec.2013.01.014.

26. T.K. Thanusha, R. Ramesh, A.S.N.A. Fazli, M.Y.M. Yunus and K. Ramesh, ARPN J. Eng. Appl. Sci., 11, 2639 (2016).

27. M. Rani, S. Gahlyan, A. Gaur and S. Maken, Chin. J. Chem. Eng., 23, 689 (2015); https://doi.org/10.1016/j.cjche.2014.12.003.

28. M. Rani and S. Maken, Korean J. Chem. Eng., 30, 1636 (2013); https://doi.org/10.1007/s11814-013-0076-X.

29. J.A. Riddick, W.B. Bunger and T.K. Sakano, Organic Solvents. Physical Properties and Methods of Purification, Wiley, New York, edn 4 (1986).
30. R. Gonzalez-Olmos, M. Iglesias, B.M.R.P. Santos and S. Mattedi, Phys. Chem. Liq., 46, 223 (2008); https://doi.org/10.1080/00319100701660411.

31. J. George and N.V. Sastry, Int. J. Thermophys., 24, 1697 (2003); https://doi.org/10.1023/B:IJOT.0000004100.28834.0a.

32. S. Gahlyan, M. Rani and S. Maken, J. Mol. Liq., 199, 42 (2014); https://doi.org/10.1016/j.molliq.2014.08.011.

33. S. Gahlyan, M. Rani and S. Maken, J. Mol. Liq., 219, 1107 (2016); https://doi.org/10.1016/j.molliq.2016.04.011.

34. M.I. Aralaguppi, C.V. Jadar and T.M. Aminabhavi, J. Chem. Eng. Data, 44, 435 (1999); https://doi.org/10.1021/je9802266.

35. M. Díaz Peña and G. Tardajos, J. Chem. Thermodyn., 10, 19 (1978); https://doi.org/10.1016/0021-9614(78)90144-1.

36. J.N. Nayak, M.I. Aralaguppi and T.M. Aminabhavi, J. Chem. Eng. Data, 48, 1152 (2003); https://doi.org/10.1021/je030107c.

37. D.C. Landaverde-Cortes, A. Estrada-Baltazar, G.A. Iglesias-Silva and K.R. Hall, J. Chem. Eng. Data, 52, 1226 (2007); https://doi.org/10.1021/je600554h.

38. T.M. Aminabhavi, V.B. Patil, M.I. Aralaguppi and H.T.S. Phayde, J. Chem. Eng. Data, 41, 521 (1996); https://doi.org/10.1021/je950279c.

39. J.C.R. Reis, I.M.S. Lampreia, Â.F.S. Santos, M.L.C.J. Moita and G. Douhéret, ChemPhysChem, 11, 3722 (2010); https://doi.org/10.1002/cphc.201000566.

40. S.K. Garg, T.S. Banipal and J.C. Ahluwalia, J. Chem. Thermodyn., 25, 57 (1993); https://doi.org/10.1006/icht.1993.1007. 